\title{
The analgesic efficacy of intravenous regional anesthesia (IVRA) with a forearm versus conventional upper arm tourniquet: a systematic review
}

\author{
Dekoninck V., Hoydonckx Y., Dubois J., Ory J.-P., Jamaer L., Stessel B. \\ Department of Anesthesiology - Intensive Care - Pain Medicine, Jessa Hospital, Hasselt, Belgium
}

\section{Background and goal of the study}

Intravenous regional anesthesia (IVRA) or Bier Block is a simple and effective anesthetic technique for surgery of the distal extremity. Traditionally, an upper arm tourniquet has been used to sequester the local anesthetic and to create a bloodless surgical field. Application of a forearm tourniquet however may offer several advantages, including a decrease to non-toxic levels of the dosage of local anesthetic required to produce an equal quality of analgesia and longer tolerance of the tourniquet. The optimal anesthetic technique for surgery of the distal extremity is still undecided. Therefore, we performed a systematic review and meta-analysis of randomized controlled trials to compare the analgesic efficacy of IVRA with a forearm versus an upper arm tourniquet in an adult population.

\section{Methods}

We performed a literature search in MEDLINE, EMBASE and Cochrane databases for relevant studies published in English, French, Spanish, German and Dutch until December 2015. Search term was "intravenous regional anesthesia". The titles and abstracts of articles identified through our search were reviewed by 2 independent authors. Reference lists of reviews and retrieved articles were reviewed for additional studies. Potentially relevant papers were retrieved in full and assessed for eligibility. Disagreements between the reviewers were resolved by a third author.

\section{Results and Discussion}

We identified 1227 records. After removing 494 duplicates, we screened 733 records of which 644 were excluded based on the title. We then read the abstracts of the remaining articles $(n=89)$ for eligibility. One article was retracted and 73 articles were excluded. After screening 15 full-text articles, 3 studies were included in the quantitative synthesis. Data from 3 studies (146 patients) were pooled for meta-analysis. In 2 of 3 studies, the dosage of local anesthetic was halved in the forearm tourniquet group. Considering the pooled effects of IVRA with forearm tourniquet on analgesic efficacy, there is no statistical difference between two groups; $\mathrm{RR}=0.99[0.94,1.04]$ with no identifiable heterogeneity $\left(\mathrm{I}^{2}=0 \%\right)$.

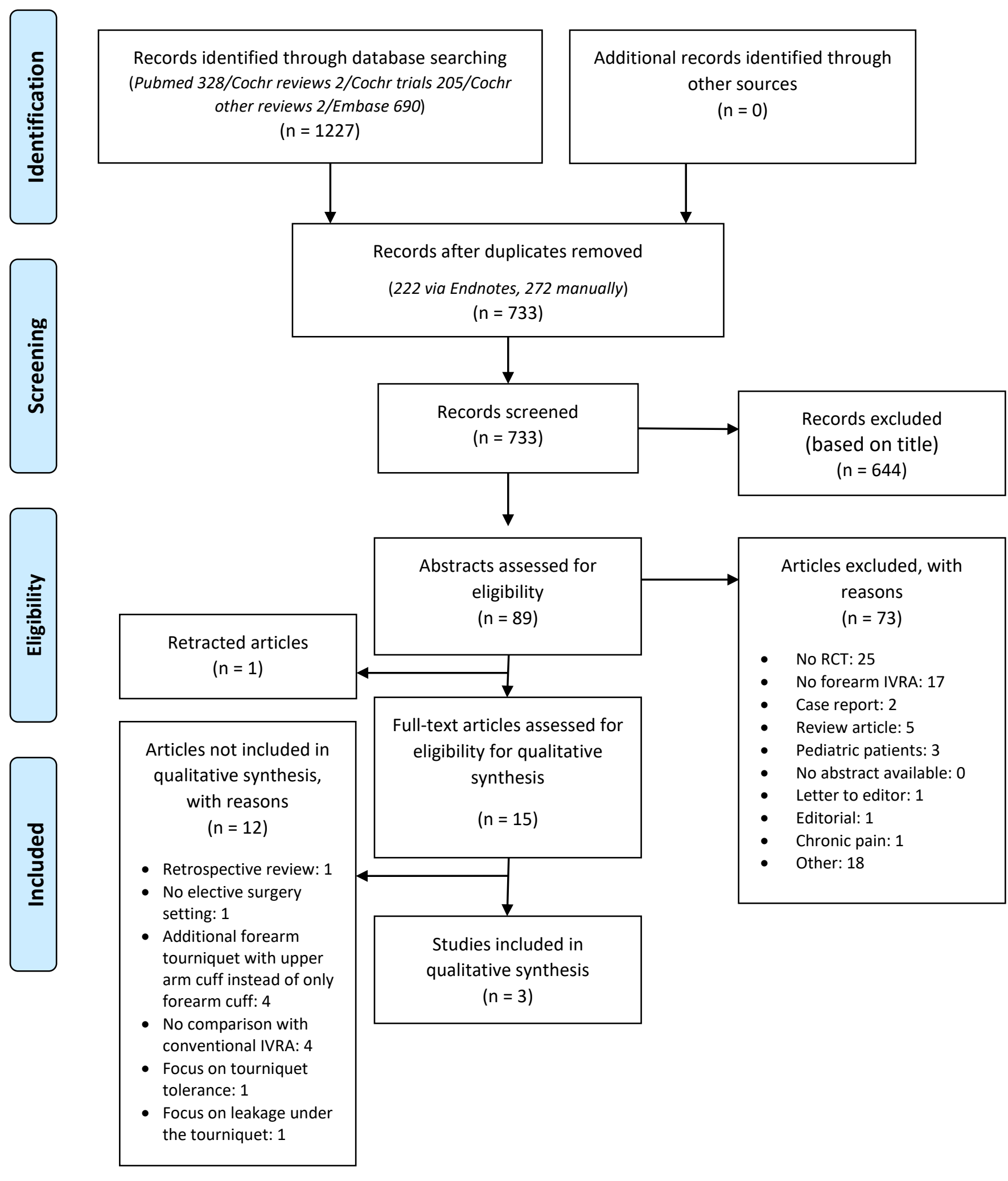

Figure 1: PRISMA flowchart depicting database search and study selection

\section{Conclusion}

This systematic review demonstrates that analgesic efficacy of IVRA with a forearm tourniquet is high and comparable with analgesic efficacy of IVRA with a conventional upper arm tourniquet. Moreover, IVRA with forearm tourniquet seems safer since the dosage of local anesthetic required, can be halved to non-toxic levels.

Figure 2: Forest plot for incidence of good anesthesia.

\begin{tabular}{|c|c|c|c|c|c|c|}
\hline \multirow[b]{2}{*}{ Study or Subgroup } & \multicolumn{2}{|c|}{ Forearm IVRA } & \multicolumn{2}{|c|}{ Upper arm IVRA } & \multirow[b]{2}{*}{ Weight } & \multirow{2}{*}{$\begin{array}{c}\text { Risk Ratio } \\
\text { M-H, Fixed, } 95 \%\end{array}$} \\
\hline & Events & Total & Events & Total & & \\
\hline Chiao et al. 2013 & 28 & 28 & 28 & 28 & $38.3 \%$ & $1.00[0.93,1$ \\
\hline Eastwood et al. 1986 & 25 & 25 & 25 & 25 & $34.2 \%$ & $1.00[0.93,1$ \\
\hline Singh et al. 2010 & 19 & 20 & 20 & 20 & $27.5 \%$ & $0.95[0.83,1$ \\
\hline Total (95\% Cl) & & 73 & & 73 & $100.0 \%$ & $0.99[0.94,1$. \\
\hline Total events & 72 & & 73 & & & \\
\hline $\begin{array}{l}\text { Heterogeneity: } \mathrm{Chi}^{2}= \\
\text { Test for overall effect: }\end{array}$ & $\begin{array}{l}55, \mathrm{df}=2 \\
=0.51(\mathrm{P}\end{array}$ & $=0.76$ & $5 ; 1^{2}=0 \%$ & & & \\
\hline
\end{tabular}

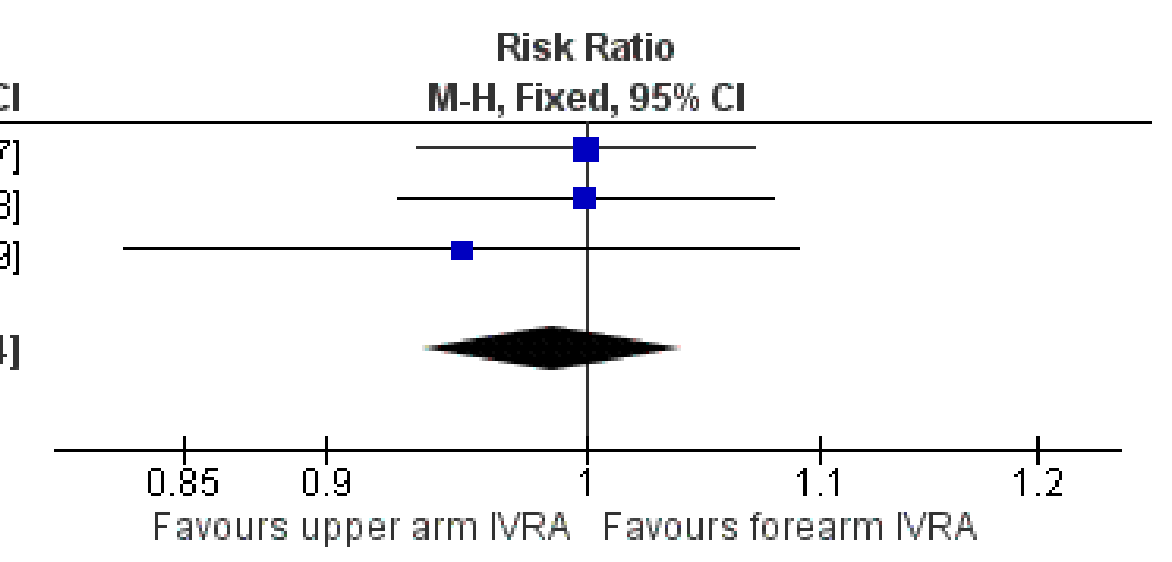

Reference

Brown EM, McGriff JT, Malinowski RW. Intravenous regional anaesthesia (Bier block): review of 20 years' experience. Can J Anaesth. 1989 